\title{
White noise excitation of road vehicle structures
}

\author{
WERNER SCHIEHLEN \\ University of Stuttgart, Stuttgart, Germany \\ e-mail: schiehlen@mechb.uni-stuttgart.de
}

\begin{abstract}
Heave and pitch motions of road vehicle structures affect the comfort and the safety of passengers. Excitation of these vertical vibrations is due to road surface roughness. Road vehicle structures are modelled as mechanical systems characterized by their inertia, damping and stiffness, and represented as state equations. This paper deals with the influence of random road profiles on the vertical dynamics of road vehicles characterized by stochastic processes. Switching from road profile displacement to road profile velocity results in white noise excitation facilitating mathematical analysis. Some fundamentals of power spectral density analysis and covariance analysis are reviewed. A quarter car model is used to show the advantages of the covariance analysis resulting immediately in standard variations characterizing the vehicle's performance.
\end{abstract}

Keywords. Road vehicles; white noise; power spectral density; covariance analysis; vehicle performance.

\section{Introduction}

Probabilistic structural dynamics and earthquake engineering are closely related to each other and they are commonly based on random vibrations and stochastic processes. Important contributions to this field are due to R N Iyengar, Indian Institute of Science, Bangalore. In his early papers he developed a non-stationary random process model for earthquake accelerograms (Iyengar \& Iyengar 1969), as well as a probabilistic response analysis approach to earthquakes (Iyengar \& Iyengar 1970). A survey on the many results achieved in this area during the following three decades was made by Iyengar (2001). However, there are also applications of stochastic processes to spacecraft structures (Iyengar \& Dash 1978), and to railway tracks (Iyengar \& Jaiswal 1995).

On the other hand, railway track unevenness and road unevenness result in random vibrations of vehicles which have to be analysed by efficient computer methods. In particular, it turns out that road unevenness can be described with good approximation by a white noise excitation process on the velocity level. Then, the corresponding vibration analysis is facilitated to such an extent that a thorough mathematical treatment is feasible resulting in better understanding of the related vehicle dynamics.

A list of symbols is given at the end of the paper

This paper is dedicated to Prof R N Iyengar of the Indian Institute of Science on the occasion of his formal retirement. 
In this paper the equations of motion and the corresponding state equations of vehicle structures are presented. Then, models for road unevenness profiles are considered and stochastic processes for vehicle excitations are introduced. Power spectral density analysis and covariance analysis are shortly reviewed. Finally, a quarter car model of road vehicles is investigated in detail and recommendations for the choice of the design parameters are given.

\section{State equations of vehicle structures}

The mechanical structures of vehicles are modelled using the multibody system approach (Schiehlen 1997). Then, the resulting equations of motion are always ordinary differential equations (ODEs). However, their form depends on the type of the multibody system. There are ideal and non-ideal systems, the first ones are characterized by applied forces and torques independent of any constraint or reaction forces respectively, while the second show such a dependency: For example, gravitational forces, spring and damper forces are independent of any reactions, while sliding friction forces and slip dependent contact forces, regularly found with tyres in vehicle dynamics, are a function of the normal or reaction forces respectively. Within the class of ideal systems, ordinary and general multibody systems are distinguished. Ordinary multibody systems are due to holonomic constraints and applied forces depending only on position and velocity quantities, and can be always represented by a system of differential equations of the second order. For non-holonomic constraints and/or general force laws we get general multibody systems (see also Eberhard \& Schiehlen 2004).

Vertical vibrations of vehicle structures rank among ordinary multibody systems. Their equations of motion read as

$$
\boldsymbol{M}(\boldsymbol{y}, t) \ddot{\boldsymbol{y}}(t)+\boldsymbol{k}(\boldsymbol{y}, \dot{\boldsymbol{y}}, t)=\boldsymbol{q}(\boldsymbol{y}, \dot{\boldsymbol{y}}, t),
$$

where $\boldsymbol{y}$ is the $f \times 1$-position vector of the generalized coordinates associated to the $f$ degrees of freedom, $\boldsymbol{M}$ is the $f \times f$-symmetric inertia matrix, $\boldsymbol{k}$ is a $f \times 1$-vector of generalized gyroscopic forces including the Coriolis and centrifugal forces as well as the gyroscopic torques, and the $f \times 1$-vector $\boldsymbol{q}$ represents generalized applied forces.

In vehicle dynamics the deviations $\tilde{\boldsymbol{y}}(t)$ from a reference motion of the vehicle $\boldsymbol{y}=\boldsymbol{y}_{R}(t)$ are often small,

$$
\boldsymbol{y}(t)=\boldsymbol{y}_{R}(t)+\tilde{\boldsymbol{y}}(t) .
$$

Then, it follows by a Taylor-series expansion under assumption of differentiable vector functions, and skipping of the second and higher order terms from (1) the linearized equations of motion (see also Popp \& Schiehlen 1993),

$$
\boldsymbol{M}(t) \ddot{\tilde{\boldsymbol{y}}}(t)+\boldsymbol{P}(t) \dot{\tilde{\boldsymbol{y}}}(t)+\boldsymbol{Q}(t) \tilde{\boldsymbol{y}}(t)=\boldsymbol{h}(t),
$$

where $\boldsymbol{M}(t)$ is the symmetric, positive definite inertia matrix while $\boldsymbol{P}(t)$ and $\boldsymbol{Q}(t)$ characterize the velocity and position dependent forces and the vector $\boldsymbol{h}(t)$ represents the external excitations. If all these matrices are time-invariant and subdivided in a symmetric and skewsymmetric part, then the equations of motion of a linear ordinary and time-invariant multibody system are,

$$
\boldsymbol{M} \ddot{\boldsymbol{y}}(t)+(\boldsymbol{D}+\boldsymbol{G}) \dot{\boldsymbol{y}}(t)+(\boldsymbol{K}+\boldsymbol{N}) \boldsymbol{y}(t)=\boldsymbol{h}(t),
$$


where $\tilde{y}$ is simply replaced by $\boldsymbol{y}$ and the $f \times f$-matrices have the properties

$$
\boldsymbol{M}=\boldsymbol{M}^{T}>\mathbf{0}, \boldsymbol{D}=\boldsymbol{D}^{t}, \boldsymbol{G}=-\boldsymbol{G}^{t}, \boldsymbol{K}=\boldsymbol{K}^{t}, \boldsymbol{N}=-\boldsymbol{N}^{t} .
$$

The physical meaning of these matrices can be identified after premultiplication of (5) from the left by $\dot{\boldsymbol{y}}^{t}$ resulting in the total time derivative of an energy expression,

$$
\begin{array}{r}
\underbrace{\dot{\boldsymbol{y}}^{t} \boldsymbol{M} \ddot{\boldsymbol{y}}}+\underbrace{\dot{\boldsymbol{y}}^{t} \boldsymbol{D} \dot{\boldsymbol{y}}}+\underbrace{\dot{\boldsymbol{y}}^{t} \boldsymbol{G} \dot{\boldsymbol{y}}}+\underbrace{\dot{\boldsymbol{y}}^{t} \boldsymbol{K} \boldsymbol{y}}+\underbrace{\dot{\boldsymbol{y}}^{t} \boldsymbol{N} \boldsymbol{y}}=\underbrace{\dot{\boldsymbol{y}}^{t} \boldsymbol{h}}, \\
T+2 R+0+\frac{\mathrm{d}}{\mathrm{d} t} U+2 S=P .
\end{array}
$$

The inertia matrix $\boldsymbol{M}$ determines the kinetic energy $T=\frac{1}{2} \dot{\boldsymbol{y}}^{t} \boldsymbol{M} \dot{\boldsymbol{y}}$ and therefore the inertia forces, from $T>0$ it follows again the positive definiteness of the inertia matrix. The damping matrix $\boldsymbol{D}$ defines via Rayleigh's dissipation function $R=\frac{1}{2} \dot{\boldsymbol{y}}^{t} \boldsymbol{D} \dot{\boldsymbol{y}}$ the damping forces, while the gyro matrix $\boldsymbol{G}$ describes the gyroscopic forces which do not change the total energy of the system. The stiffness matrix determines the potential energy $U=\frac{1}{2} \boldsymbol{y}^{t} \boldsymbol{K} \boldsymbol{y}$ and, therefore, the conservative position forces, while the matrix $\boldsymbol{N}$ identifies the circulatory forces also known as non-conservative position forces. Furthermore, $P$ describes the power of the external excitation forces. For $\boldsymbol{D}=\mathbf{0}, \boldsymbol{N}=\mathbf{0}$ and $\boldsymbol{h}=0$ the multibody system is conservative, i.e., the total energy is constant for all motions,

$$
T+U=\text { const. }
$$

The matrix properties (5) allow often us to check the equations of motion with respect to the physical phenomena involved.

The generation of equations of motion for large multibody systems is a nontrivial task requiring numerous steps during the evaluation of the fundamental relations. Beginning with the space age in the middle of the 1960s, the generation of equations of motion are more formalized. The resulting formalisms have been used for the development of computer codes for multibody systems, they are the basis of computational multibody dynamics. Twenty-five years later there were known 20 formalisms described in the Multibody System Handbook (Schiehlen 1990), many of them are used today. Furthermore, non-recursive and recursive formalisms are distinguished. Recursive formalisms make use of special topology properties of multibody systems (see e.g. Schiehlen 2005).

For the time integration of ordinary multibody systems the mass matrix in the equations of motion (1) has to be inverted, which is numerically costly for systems with many degrees of freedom,

$$
\ddot{\boldsymbol{y}}(t)=\boldsymbol{M}^{-1}(\boldsymbol{y}, t)[\boldsymbol{q}(\boldsymbol{y}, \dot{\boldsymbol{y}}, t)-\boldsymbol{k}(\boldsymbol{y}, \dot{\boldsymbol{y}}, t)] .
$$

Recursive algorithms avoid this matrix inversion. The fundamental requirement, however, is a chain or tree topology of the multibody system that is often found in vehicle dynamics.

Moreover, the equations of motion (1) which are vector differential equations of the second order have to be supplemented by the initial conditions for the generalized positions and velocities,

$$
\boldsymbol{y}(0)=\boldsymbol{y}_{0}, \dot{\boldsymbol{y}}(0)=\dot{\boldsymbol{y}}_{0}
$$

The dynamical behaviour of vehicles can be described more consistently by the state space approach widely used in control and system theory (see e.g. De Russo et al 1997). For this 
purpose, the vehicle's $n \times 1$-state vector is composed by its position and velocity vector as,

$$
\boldsymbol{x}(t)=\left[\begin{array}{c}
\boldsymbol{y}(t) \\
\dot{\boldsymbol{y}}(t)
\end{array}\right],
$$

where for ordinary multibody systems it yields $n=2 f$ with the number $f$ denoting the degrees of freedom.

Based on the state vector (11) the equations of motion are simply transformed into the state equations,

$$
\begin{aligned}
\dot{\boldsymbol{y}}(t) & =\dot{\boldsymbol{y}}(t), \\
\underbrace{\ddot{\boldsymbol{y}}(t)}_{\dot{\boldsymbol{x}}(t)} & =\underbrace{\boldsymbol{M}^{-1}(\boldsymbol{y}, t)[\boldsymbol{q}(\boldsymbol{y}, \dot{\boldsymbol{y}}, t)-\boldsymbol{k}(\boldsymbol{y}, \dot{\boldsymbol{y}}, t)]}_{\boldsymbol{a}(\boldsymbol{x}, t)},
\end{aligned}
$$

where the nonlinear $n \times 1$-vector function $\boldsymbol{a}(\boldsymbol{x}, t)$ appears. For vehicles with small linear deviations from a reference motion, it remains

$$
\dot{\boldsymbol{x}}(t)=\boldsymbol{A x}(t)+\boldsymbol{B} \boldsymbol{u}(t),
$$

where

$$
\boldsymbol{A}=\left[\begin{array}{cc}
\mathbf{0} & \boldsymbol{E} \\
-\boldsymbol{M}^{-1}(\boldsymbol{K}+\boldsymbol{N}) & -\boldsymbol{M}^{-1}(\boldsymbol{D}+\boldsymbol{G})
\end{array}\right],
$$

is the $n \times n$-system matrix and $\boldsymbol{B}$ represents the $n \times r$-input matrix of the $r$ excitations and controls acting on the vehicle and summarized in the $r \times 1$-vector $\boldsymbol{u}(t)$. According to (10) the state equations have to be supplemented by the initial conditions

$$
x(0)=x_{0} .
$$

Since the state equations are vector differential equations of first order there is one $n \times 1$ vector (15) sufficient to describe the initial conditions uniquely. However, the vector (15) is of twice the order as the vectors in (10).

\section{Models of road unevenness profiles}

The road roughness profiles $Z(x)$ represent stochastic processes depending on the spatial road coordinate $x$ of the travelling vehicle (see e.g. Popp 1982). The evaluation and processing of numerous measurement data of road roughness profiles $Z(x)$ shows a common tendency allowing some standardization (see ISO-8608 1995; von Scheidt 1997, Mucka 2004). The one-sided power spectral density (PSD) is a simple but often used road roughness model reading as

$$
\Phi_{Z}(\Omega)=\Phi_{0}\left(\Omega_{0} / \Omega\right)^{w}, 0<\Omega_{I} \leq \Omega \leq \Omega_{\Pi}<\infty,
$$

where the frequency range is bounded. Here, $\Omega_{0}[\mathrm{rad} / \mathrm{m}]$ denotes a standardized spatial circular frequency (wave number), $\Phi_{0}=\Phi_{Z}\left(\Omega_{0}\right)\left[\mathrm{m}^{2} /(\mathrm{rad} / \mathrm{m})\right]$ characterizes the roughness as 


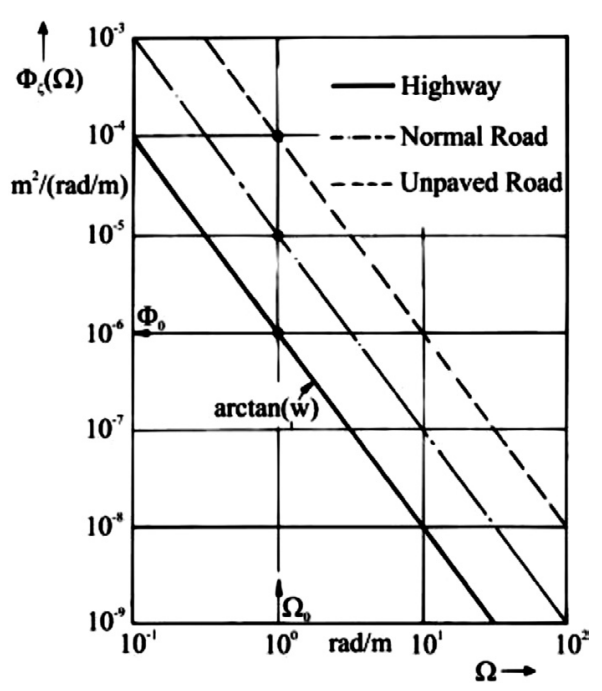

Figure 1. Power spectral density of roads with a waviness of $w=2$.

a function of the spatial frequencies $\Omega_{0}$ and can be considered as roughness level, and $w$ is called waviness and indicates whether the road has more long wavelengths ( $w$ large) or short wavelengths ( $w$ small). For the wavelength $\lambda$, the relation $\lambda=2 \pi / \Omega$ holds. The waviness $w$ of road profiles is found within the range $1.75<w<2.25$ with $w=2$ as a good average value. Equation (16) is usually plotted in a $\Phi, \Omega$-diagram with double logarithmic scale and resulting in decreasing straight lines with slopes $(-2 w)$, figure 1 .

A similar but more complicated model gives (Dodds \& Robson 1973),

$$
\Phi_{Z}(\Omega)=\left\{\begin{array}{lll}
\Phi_{0}\left(\frac{\Omega_{0}}{\Omega}\right)^{w 1} & & 0<\Omega_{I} \leq \Omega \leq \Omega_{0}, \\
& \text { for } & \\
\Phi_{0}\left(\frac{\Omega_{0}}{\Omega}\right)^{w 2} & \Omega_{0} \leq \Omega \leq \Omega_{I I}<\infty,
\end{array}\right.
$$

where different values $w_{1}, w_{2}, w_{1}<w_{2}$ of waviness occur in two frequency ranges.

The road roughness models (16) and (17) are approximations to measured PSD in a certain frequency range $0<\Omega_{I}<\Omega<\Omega_{I I}<\infty$. In the limit case $\Omega \rightarrow \infty$ both models result in infinite values of the PSD, $\Phi_{Z}(0) \rightarrow \infty$, and, thus, in infinite variances, $P_{Z} \rightarrow \infty$. To avoid this unrealistic case, extended road roughness models have been suggested to be valid in the whole frequency range. These models represent coloured noise processes characterised by the first and second order PSDs as

$$
\begin{aligned}
\text { I : } \quad \Phi_{Z I}(\Omega)=\frac{2 \alpha \sigma^{2}}{\pi} \frac{1}{\alpha^{2}+\Omega^{2}}, \quad 0 \leq \Omega<\infty \\
\text { II : } \quad \Phi_{Z I I}(\Omega)=\frac{2 \alpha \sigma^{2}}{\pi} \frac{\Omega^{2}+\alpha^{2}+\beta^{2}}{\left(\Omega^{2}-\alpha^{2}-\beta^{2}\right)^{2}+4 \alpha^{2} \Omega^{2}}, \quad 0 \leq \Omega<\infty
\end{aligned}
$$

where $\alpha, \beta$ and $\sigma^{2}$ are positive constants. The corresponding correlation functions can be calculated using an inverse Fourier transform,

$$
R_{Z}(\xi)=\int_{0}^{\infty} \Phi_{Z}(\Omega) \cos \Omega \xi \mathrm{d} \xi,
$$


resulting in

(I) $\quad R_{Z}(\xi)=\sigma^{2} e^{-\alpha|\xi|}$,

(II) $\quad R_{Z}(\xi)=\sigma^{2} e^{-\alpha|\xi|} \cos \beta \xi$,

where $\sigma^{2}=R_{Z}(\xi=0)$ denotes the finite variance of the road roughness profile and $\xi$ means the correlation distance. For $\Omega \rightarrow \infty$ both PSDs (18), (19) decay according to $\Phi \approx 1 / \Omega^{2}$. Thus, they approximate the measured road roughness profiles fairly well.

From the models of the road roughness profiles $Z(x)$ shown above, corresponding models of the vehicle excitation $\zeta(t)$ in time domain can be derived. Here, permanent road contact is assumed and, in a first step, only one contact point is considered. The transformation from space to time domain, $Z(x) \rightarrow \zeta(t)$ is performed considering the vehicle speed $v(t)$,

$$
\mathrm{d} x(t)=v(t) \mathrm{d} t, x(t)=x\left(t_{0}\right)+\int_{t_{0}}^{t} v(\tau) \mathrm{d} \tau .
$$

From the road roughness profiles stationary in space domain, it generally follows nonstationary vehicle excitation processes in time domain if the vehicle speed $v(t)$ is time varying, which will not be considered here. Throughout this paper, a constant vehicle speed $v=$ const is assumed resulting in stationary vehicle excitation processes. For $t_{0}=0$ and $x\left(t_{0}\right)=0$ from (23) it follows

$$
x(t)=v t, \xi=v \tau, \omega=v \Omega, v=\text { const. }
$$

Here, $\tau$ means the correlation time corresponding to the correlation width $\xi$ and $\omega$ with unit $\mathrm{rad} / \mathrm{s}$ denotes the temporal circular frequency. Since the variances $R_{Z}(0)$ of road profiles $Z(x)$ and vehicle excitations $\zeta(t)$ must be equal, (20) yields $\Phi_{Z}(\Omega) \mathrm{d} \Omega=\Phi_{\zeta}(\omega) \mathrm{d} \omega$ that with (24) results in the PSD $\Phi_{\zeta}(\omega)\left[\mathrm{m}^{2} /(\mathrm{rad} / \mathrm{s})\right]$ of the vehicle excitation

$$
\Phi_{\zeta}(\omega)=\frac{1}{v} \Phi_{Z}\left(\Omega=\frac{\omega}{v}\right) .
$$

For example, from the road roughness model (16) with the waviness $w=2$ it follows,

$$
\tilde{\Phi}_{\zeta}(\omega)=(1 / v) \tilde{\Phi}_{0}\left(v \Omega_{0} / \omega\right)^{2}=v \tilde{\Phi}_{0}\left(\Omega_{0} / \omega\right)^{2} .
$$

Until now only stochastic excitation processes $\zeta(t)$ of the vertical road displacements are considered. But the differentiated processes $\dot{\zeta}(t)$ and $\ddot{\zeta}(t)$ of vertical road velocity and acceleration respectively, can also be regarded. The corresponding PSDs read,

$$
\Phi_{\dot{\zeta}}(\omega)=\omega^{2} \Phi_{\zeta}(\omega), \Phi_{\ddot{\zeta}}(\omega)=\omega^{4} \Phi_{\zeta}(\omega) .
$$

Equation (27) applied to (26), for example, yields a white noise process for the vehicle excitation velocity $\dot{\zeta}(t)$ characterised by the PSD

$$
\tilde{\Phi}_{\dot{\zeta}}(\omega)=\omega^{2} v \tilde{\Phi}_{0}\left(\Omega_{0} / \omega\right)^{2}=v \tilde{\Phi}_{0} \Omega_{0}^{2}=\text { const. }
$$

The corresponding correlation function reads

$$
R_{\dot{\zeta}}(\tau)=q_{\dot{\zeta}} \delta(\tau), q_{\dot{\zeta}}=\pi v \tilde{\Phi}_{0} \Omega_{0}^{2},
$$




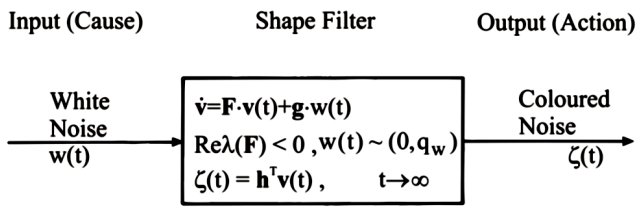

Figure 2. Shape filter for random road unevenness modelling.

where $q_{\dot{\zeta}}$ denotes the noise intensity of $\dot{\zeta}(t)$ and $\delta(\tau)$ is the Dirac distribution. Assuming the waviness $w=4$ it follows similarly a white noise process for the vehicle excitation acceleration $\ddot{\zeta}(t)$,

$$
\begin{aligned}
& \approx_{\breve{\zeta}}(\omega)=\omega^{4} v \widetilde{\widetilde{\Phi}}_{0}\left(\frac{\Omega_{0}}{\omega}\right)^{4}=v \widetilde{\widetilde{\Phi}}_{0} \Omega_{0}^{4}=\mathrm{const}, \\
& R_{\ddot{\zeta}}(\omega)=q_{\ddot{\zeta}}^{\delta}(\tau), q_{\ddot{\zeta}}=\pi v \widetilde{\widetilde{\Phi}}_{0} \Omega_{0}^{4} .
\end{aligned}
$$

The white noise processes are characterised by infinite variances and, thus, they are not realistic. On the other hand, assuming white noise vehicle excitations (28)-(31), the computation effort for determining vehicle random vibrations can be reduced considerably. Therefore, the white noise velocity excitation of road vehicles is a useful engineering approximation.

More complex models of vehicle excitations $\zeta(t)$ are based on (18) to (22) representing stationary Gaussian coloured noise processes. These processes can be generated as steady state solution of a system of linear time invariant ordinary differential equations (ODEs) excited by white noise $w(t)$. These ODEs change - graphically speaking - the shape of the PSD or the corresponding correlation function, thus, they are called shape filter, figure 2 . It holds generally that the properties - stationarity, normal Gaussian distribution and ergodicity - will be transferred from the system input to the system output if the shape filter is designed by a system of linear time invariant ODEs. Thus, an appropriate shape filter is given as,

$$
\begin{aligned}
& \zeta(t)=\boldsymbol{h}^{T} v(t), \\
& \dot{\boldsymbol{v}}(t)=\boldsymbol{F} \boldsymbol{v}(t)+\boldsymbol{g} w(t), \operatorname{Re} \lambda(\boldsymbol{F})<0, \quad w(t)\left(0, q_{w}\right) .
\end{aligned}
$$

In the steady state, the coloured noise process $\varsigma(t)$ is generated by superposition of the state variables $v_{i}(t), i=1(1) m$, that are combined to the $m \times 1$-state vector $\boldsymbol{v}$ according to (32). This state vector $\boldsymbol{v}$ is the output of the system (33) of ODEs, the system matrix $\boldsymbol{F}$ is asymptotically stable, and the excitation is given by white noise $w(t)$ with zero mean and intensity $q_{w}$. The quantities $\boldsymbol{F}, \boldsymbol{g}$ and $\boldsymbol{h}$ characterize the shape filter completely. For the models (18) to (22) these quantities read

$$
\begin{aligned}
\text { I }: \quad \boldsymbol{F}= & -\alpha v, \boldsymbol{g}=g, \boldsymbol{h}=1 \quad\left(g^{2} q_{w}=2 \alpha v \sigma^{2}\right) \\
\mathrm{II}: \quad \boldsymbol{F}= & {\left[\begin{array}{cc}
0 & 1 \\
-\left(\alpha^{2}+\beta^{2}\right) v^{2} & -2 \alpha v
\end{array}\right], \boldsymbol{g}=g\left[\begin{array}{l}
0 \\
1
\end{array}\right], \boldsymbol{h}=\left[\begin{array}{c}
v\left(\alpha^{2}+\beta^{2}\right)^{1 / 2} \\
1
\end{array}\right], } \\
& \left(g^{2} q_{w}=2 \alpha v \sigma^{2}\right) .
\end{aligned}
$$

Since the product $g^{2} q_{w}$ of the shape filter quantities follows from a product of model parameters, there is a possibility of choice. If $g=1$ is chosen, for example, then for both shape filters 
the intensity $q_{w}$ of the white noise excitation is $q_{w}=2 \alpha v \sigma^{2}$. This shows that the intensity of the vehicle excitation grows proportional to the vehicle speed $v$.

In general, the shape filter quantities should be determined directly from measured PSDs $\Phi_{\zeta}(\Omega)$ rather than from analytical approximations like shown here. Then, the order $m$ of the filter has to be chosen properly. For $m=2$ this procedure is extensively described by Mueller et al (1980). Further, for multi-axle vehicle the excitations are characterized by time delay as shown, e.g., by Popp \& Mueller (1979).

\section{Vehicle random vibration analysis}

The vehicle structure including the road excitation $\zeta(t)$ can be described by the linear state equation,

$$
\dot{\boldsymbol{x}}(t)=\boldsymbol{A x}(t)+\boldsymbol{B} \boldsymbol{u}(\zeta, t),
$$

where $\boldsymbol{x}$ is the state vector, $\boldsymbol{u}$ characterizes the excitation by the road, $\boldsymbol{A}$ denotes the state matrix and $\boldsymbol{B}$ the input matrix. As a criterion for ride comfort, the acceleration $a(t)$ at a seat position is considered which can be composed of the state and excitation quantities of the vehicle structure,

$$
a(t)=\boldsymbol{c}^{T} \boldsymbol{x}(t)+\boldsymbol{d}^{T} \boldsymbol{u}(\zeta, t),
$$

where $\boldsymbol{c}$ is a weighting vector of the states and $\boldsymbol{d}$ a weighting vector of the excitations.

Moreover, the state equations (36) can be extended by the shape filters (32), (33) resulting in

$$
\begin{aligned}
& \underbrace{\left[\begin{array}{c}
\dot{\boldsymbol{x}} \\
\dot{\boldsymbol{y}}
\end{array}\right]}=\underbrace{\left[\begin{array}{cc}
\boldsymbol{A} & \boldsymbol{B} \boldsymbol{H} \\
\mathbf{0} & \boldsymbol{F}
\end{array}\right]}\left[\begin{array}{l}
\boldsymbol{x} \\
\boldsymbol{v}
\end{array}\right]+\underbrace{\left[\begin{array}{l}
\mathbf{0} \\
\boldsymbol{g}
\end{array}\right]} \underbrace{w(t)}, \\
& \tilde{\boldsymbol{x}}=\tilde{\boldsymbol{A}} \tilde{\boldsymbol{x}}+\tilde{\boldsymbol{B}} \tilde{\boldsymbol{u}} .
\end{aligned}
$$

The extended system (38) has exactly the same structure as the vehicle system (36), so that system (36) is considered subsequently without loss of generality. The required computational methods can be subdivided into methods that are described in the frequency or time domain using power spectral density analysis or covariance analysis respectively.

Assuming that $\boldsymbol{x}(t)=\mathbf{0}$ is an asymptotically stable equilibrium position, from $E\{\boldsymbol{w}(t)\}=\mathbf{0}$ it follows that $E\{\boldsymbol{x}(t)\}=\mathbf{0}$, i. e. the mean value of the state vector vanishes in steady state. Thus, as an essential goal it remains to calculate the characterizing variances, e. g. the variance $\sigma_{a}^{2}=E\left\{a^{2}\right\}$ of the acceleration (37) describing ride comfort.

The two-sided power spectral density (PSD) is a characteristic quantity of a random process in the frequency domain. In particular, for linear systems the PSD of the input and output processes are related to each other by the system's frequency response matrix. However, we should keep in mind that the ride comfort and safety criteria depend primarily on variances and not on PSD's.

The $n \times n$ - PSD-matrix $\boldsymbol{S}_{x}(\omega)$ of the entire vehicle system described by the state equation (36) with $\boldsymbol{A}=\mathbf{c o n s t}$ and $\boldsymbol{B}=\mathbf{c o n s t}$ follows for the white noise process $\boldsymbol{w}(t) \sim\left(\mathbf{0}, \boldsymbol{Q}_{w}\right)$ as,

$$
\boldsymbol{S}_{x}(\omega)=(i \omega \boldsymbol{E}-\boldsymbol{A})^{-1} \boldsymbol{B} \boldsymbol{Q}_{w} \boldsymbol{B}^{T}(-i \omega \boldsymbol{E}-\boldsymbol{A})^{-T} .
$$


Thus, the PSD-matrix $\boldsymbol{S}_{x}(\omega)$ is obtained from the input intensity matrix $\boldsymbol{Q}_{w}$ by matrix multiplications with the frequency response matrix $\boldsymbol{F}_{x}=(i \omega \boldsymbol{E}-\boldsymbol{A})^{-1}$. Since the state vector $\boldsymbol{x}(t)$ and the system matrix $\boldsymbol{A}$ can often be partitioned it is possible to get as intermediate results the PSD-matrices of the road excitation, the suspension system and the chassis respectively. This may reduce the numerical amount to some extent.

The scalar PSD $S_{a}(\omega)$ of the scalar acceleration $a(t)$, based on (37) reads

$$
S_{a}(\omega)=\left(\boldsymbol{c}^{T} \boldsymbol{F}_{x}(\omega) \boldsymbol{B}+\boldsymbol{d}^{T}\right) \boldsymbol{Q}_{w}\left(\boldsymbol{B}^{T} \boldsymbol{F}_{x}^{T}(-\omega) \boldsymbol{c}+\boldsymbol{d}\right),
$$

where the $n \times n$-frequency response matrix $\boldsymbol{F}_{x}$ has been used again. Equations (39) and (40) show that the PSD is a quadratic expression; i.e., the PSD of a sum of quantities leads to more complex terms in the result.

The variance follows from the PSD by integration over an infinite interval,

$$
\sigma_{a}^{2}=\int_{-\infty}^{\infty} S_{a}(\omega) \mathrm{d} \omega=\int_{0}^{\infty} \Phi_{a}(\omega) \mathrm{d} \omega
$$

For convenience, in (41) the single-sided PSD $\Phi_{a}(\omega)$ with $\Phi_{a}(\omega)=2 S_{a}(\omega)$ has been introduced as well.

The numerical integration of the PSD (41) is generally not difficult. However, we have to deal with complex matrices, many approximation points and a very large integration interval leading to numerical errors and considerable computation times. Integration (41) can be avoided by applying the covariance analysis as shown in the following.

In contrast to spectral analysis, the covariance analysis directly yields the variances that are required for the assessment of ride comfort and ride safety. The covariance matrix of the entire vehicle system follows from a linear algebraic equation, the so-called Lyapunov matrix equation, and no integrations are required. An essential prerequisite of the covariance analysis is a white noise input process. This can always be provided by modelling the random vehicle excitation by means of a shape filter.

The Lyapunov matrix equation based on the state equation (36) of the entire vehicle system is

$$
\boldsymbol{A} \boldsymbol{P}_{x}+\boldsymbol{P}_{x} \boldsymbol{A}^{T}+\boldsymbol{B} \boldsymbol{Q}_{w} \boldsymbol{B}^{T}=\mathbf{0},
$$

where $\boldsymbol{P}_{x}=E\left\{\boldsymbol{x} \boldsymbol{x}^{T}\right\}$ denotes the symmetric $n \times n$ - covariance matrix and $\boldsymbol{Q}_{w}$ is the $r \times r$ intensity matrix of the white noise input. An extensive derivation of (42) can be found in Mueller \& Schiehlen (1985). Stable numerical procedures to solve the Lyapunov matrix equation are given in Smith (1968) and Kreisselmeier (1972). As a result, we get the variances of all state variables of the system. Since the computation of the ride comfort requires the variance of the acceleration, covariance analysis has to be applied to the extended system (38). The covariance matrix of the extended state vector $\tilde{\boldsymbol{x}}$ is

$$
\boldsymbol{P}_{\tilde{x}}=\left[\begin{array}{cc}
\boldsymbol{P}_{x} & \boldsymbol{P}_{x v} \\
\boldsymbol{P}_{v x} & \boldsymbol{P}_{v}
\end{array}\right],
$$

where $\boldsymbol{P}_{v x}=\boldsymbol{P}_{x \nu}^{\boldsymbol{T}}$ holds. From (37) it follows immediately that

$$
\sigma_{a}^{2}=c^{T} \boldsymbol{P}_{x} c+2 c^{T} \boldsymbol{P}_{x v} H d+d^{T} \boldsymbol{H}^{T} \boldsymbol{P}_{v} H d .
$$

This is the relation corresponding to (41) but requires only linear matrix calculus. 


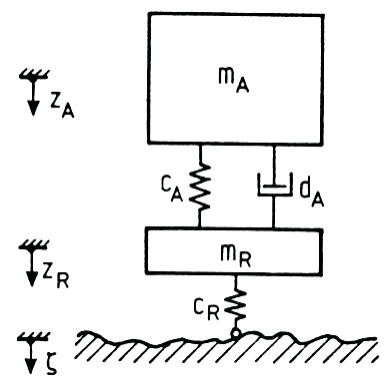

Figure 3. Quarter car model.

\section{Random excitation of a quarter car model}

For the design of vehicle suspensions, the random road unevenness profiles have to be taken into account. There are two main tasks of a vehicle suspension. On the one hand, the vehicle body has to be carried at constant height without vibrational accelerations, while on the other hand the vehicle wheels should follow the uneven profile of the road without any delay resulting in a constant wheel load. Both requirements are inconsistent with each other, and, therefore, the problem may be solved by frequency decoupling. Then, the eigenfrequency of the body should be as low as possible while the eigenfrequency of the wheels has to be as high as possible. However, there are design constraints which restrain the frequency decoupling, like the limited relative motion space between of the wheels and the body. This means that an optimal design has to consider the engineering constraints, and the design process has to include the vehicle structure and the randomly uneven road.

The fundamentals of vehicle suspensions are presented for a quarter car model, figure 3 , which offers frequency decoupling by two degrees of freedom and includes random excitation of by road unevenness. With respect to the equilibrium condition the linear equations of motion are,

$$
\left[\begin{array}{cc}
m_{A} & 0 \\
0 & m_{R}
\end{array}\right]\left[\begin{array}{c}
\ddot{z}_{A} \\
\ddot{z}_{R}
\end{array}\right]+\left[\begin{array}{cc}
d_{A} & -d_{A} \\
-d_{A} & d_{A}
\end{array}\right]\left[\begin{array}{c}
\dot{z}_{A} \\
\dot{z}_{R}
\end{array}\right]+\left[\begin{array}{cc}
c_{A} & -c_{A} \\
-c_{A} & c_{A}+c_{R}
\end{array}\right]\left[\begin{array}{c}
z_{A} \\
z_{R}
\end{array}\right]=\left[\begin{array}{c}
0 \\
c_{R}
\end{array}\right] \zeta(t)
$$

where $m_{A}$ is the body mass, $m_{R}$ is the generalized mass of all wheels and $d_{A}, c_{A}$ and $c_{R}$ represent the generalized damping coefficient, body spring coefficient and tyre spring coefficient respectively of all wheels. As excitation a white velocity noise with intensity $q$ is assumed,

$$
\dot{\zeta}(t) \sim(0, q) .
$$

Using the abbreviations

$$
a=\frac{c_{A}}{m_{A}}, b=\frac{c_{A}}{m_{R}}, c=\frac{c_{R}}{m_{R}}, d=\frac{d_{A}}{m_{A}}, e=\frac{d_{A}}{m_{R}}
$$


we gets from (45) the state equations which have been differentiated once to obtain an velocity excitation of the vehicle,

$$
\underbrace{\left[\begin{array}{c}
\ddot{z}_{A} \\
\ddot{z}_{R} \\
\dddot{z}_{A} \\
\dddot{z}_{R}
\end{array}\right]}_{\ddot{\boldsymbol{x}}=\boldsymbol{A} \dot{\boldsymbol{x}}+\boldsymbol{B} w(t)}=\underbrace{\left[\begin{array}{cccc}
0 & 0 & 1 & 0 \\
0 & 0 & 0 & 1 \\
-a & a & -d & d \\
b & -b-c & e & -e
\end{array}\right]} \underbrace{\left[\begin{array}{c}
\dot{z}_{A} \\
\dot{z}_{R} \\
\ddot{z}_{A} \\
\ddot{z}_{R}
\end{array}\right]}+\underbrace{\left[\begin{array}{l}
0 \\
0 \\
0 \\
c
\end{array}\right]} \dot{\underbrace{\dot{\zeta}(t)}} .
$$

For the assessment of the vehicles performance, driving comfort and driving safety are used. This yields for the vertical acceleration,

$$
a_{A}=\ddot{z}_{A}=\left[\begin{array}{llll}
0 & 0 & 1 & 0
\end{array}\right] \dot{\boldsymbol{x}}=\boldsymbol{c}^{T} \dot{\boldsymbol{x}}
$$

and for the dynamical tyre load,

$$
f=c_{R}\left(\zeta-z_{R}\right)=m_{A} \ddot{z}_{A}+m_{R} \ddot{z}_{R}=\left[\begin{array}{llll}
0 & 0 & m_{A} & m_{R}
\end{array}\right] \dot{\boldsymbol{x}}=\boldsymbol{e}^{T} \dot{\boldsymbol{x}},
$$

where the $4 \times 1$-weighting vectors are introduced. Then, it remains for the standard deviations of acceleration and wheel load that

$$
\sigma_{a}^{2}=c^{T} \boldsymbol{P} \boldsymbol{c}=\boldsymbol{P}_{33},
$$

and

$$
\sigma_{f}^{2}=\boldsymbol{e}^{T} \boldsymbol{P e}=m_{A}^{2} P_{33}+2 m_{A} m_{R} P_{34}+m_{R}^{2} P_{44},
$$

with the stationary $4 \times 4$-covariance matrix,

$$
\boldsymbol{P}=E\left\{\dot{\boldsymbol{x}}(t) \dot{\boldsymbol{x}}^{T}(t)\right\}=\text { const. }
$$

The problem is reduced now to the evaluation of the covariance matrix (53) of the system (48). For this purpose the covariance analysis is most adequate, offering analytical solutions for low order systems in contrast to the power spectral density approach. The Lyapunov matrix equation (42) is now

$$
\boldsymbol{A P}+\boldsymbol{P} \boldsymbol{A}^{T}+\boldsymbol{B q} \boldsymbol{B}^{T}=\mathbf{0} .
$$

For the solution, a matrix polynomial is applied as outlined by Mueller \& Schiehlen (1985). This polynomial for $n=4$ is as follows,

$$
\boldsymbol{P}=\frac{1}{2 \operatorname{det} \boldsymbol{H}} \sum_{k=o}^{3} H_{k, k+1} \sum_{m=0}^{2 k}(-1)^{m} \boldsymbol{A}_{\boldsymbol{m}} \boldsymbol{Q} \boldsymbol{A}_{2 m-k}^{T},
$$

where

$$
\boldsymbol{H}=\left[\begin{array}{cccc}
a_{1} & 1 & 0 & 0 \\
a_{3} & a_{2} & a_{1} & 1 \\
0 & a_{4} & a_{3} & a_{2} \\
0 & 0 & 0 & a_{4}
\end{array}\right]
$$


is the $4 \times 4$-Hurwitz matrix also known from stability theory and $H_{k+1,1}$ are the corresponding scalar cofactors. Further,

$$
a_{i}, i=1(1) 4,
$$

mean the characteristic coefficients of the system matrix $\boldsymbol{A}$. In addition, the $4 \times 4$-auxiliary matrices are defined by

$$
\boldsymbol{A}_{\boldsymbol{m}}=\boldsymbol{A} \boldsymbol{A}_{m-1}+a_{m} \boldsymbol{E}, m=0(1) 6,
$$

where it yields $\boldsymbol{A}_{p}=\mathbf{0}, p=4(1) 6$. The $4 \times 4$-intensity matrix reads as

$$
Q=q \boldsymbol{B} \boldsymbol{B}^{T} .
$$

Some intermediate results are listed for the following quantities:

Characteristic equation

$$
\operatorname{det}(\lambda \boldsymbol{E}-\boldsymbol{A})=\lambda^{4}+(d+e) \lambda^{3}+(a+b+c) \lambda^{2}+c d \lambda+a c=0 .
$$

Determinant of Hurwitz matrix

$$
\operatorname{det} H=a_{4} H_{3}=a c\left(c^{2} d e\right) .
$$

Cofactors of Hurwitz matrix

$$
\left.\begin{array}{l}
H_{11}=a_{4}\left(a_{2} a_{3}-a_{1} a_{4}\right)=a c^{3} d, \\
H_{21}=-a_{3} a_{4}=-a c^{2} d, \\
H_{31}=a_{1} a_{4}=a c(d+e), \\
H_{41}=-\left(a_{1} a_{2}-a_{3}\right)=-(a+b)(d+e)-c e .
\end{array}\right\}
$$

Auxiliary matrices multiplied by $4 \times 1$-matrix $\mathbf{B}$

$$
\begin{aligned}
& \boldsymbol{A}_{0} \boldsymbol{B}=\boldsymbol{B}=c\left[\begin{array}{llll}
0 & 0 & 0 & 1
\end{array}\right]^{T}, \\
& \boldsymbol{A}_{1} \boldsymbol{B}=\boldsymbol{A} \boldsymbol{B}+a_{1} \boldsymbol{B}=c\left[\begin{array}{llll}
0 & 1 & d & d
\end{array}\right]^{T}, \\
& \boldsymbol{A}_{2} \boldsymbol{B}=\boldsymbol{A} \boldsymbol{A}_{1} \boldsymbol{B}+a_{2} \boldsymbol{B}=c\left[\begin{array}{llll}
d & d & a & a
\end{array}\right]^{T}, \\
& \boldsymbol{A}_{3} \boldsymbol{B}=\boldsymbol{A} \boldsymbol{A}_{2} \boldsymbol{B}+a_{3} \boldsymbol{B}=c\left[\begin{array}{llll}
a & a & 0 & 0
\end{array}\right]^{T}, \\
& \boldsymbol{A}_{4} \boldsymbol{B}=0 .
\end{aligned}
$$

Then, the elements of the covariance matrix required for the assessments (51) and (52) are easily found as

$$
\begin{aligned}
& P_{33}=(q / 2 d e)\left[c d^{3}+a^{2}(d+e)\right], \\
& P_{34}=(q / 2 d e)\left[c d\left(d^{2}-a\right)+a^{2}(d+e)\right], \\
& P_{44}=(q / 2 d e)\left[(a-c)^{2} d+c d^{3}+a^{2} e\right] .
\end{aligned}
$$


Inserting (64) to (66) in (51) and (52), and eliminating the abbreviations (47), provide, the final results

$$
\begin{aligned}
\sigma_{a}^{2}= & \frac{q}{2}\left[\frac{c_{R} d_{A}}{m_{A}^{2}}+\frac{c_{A}^{2}\left(m_{A}+m_{R}\right)}{d_{A} m_{A}^{2}}\right], \\
\sigma_{f}^{2}= & \frac{q}{2}\left[\left(1+\frac{m_{R}}{m_{A}}\right)^{3} \frac{c_{A}^{2} m_{A}}{d_{A}}+\left(1+\frac{m_{R}}{m_{A}}\right)^{2} c_{R} d_{A}\right. \\
& \left.-2\left(1+\frac{m_{R}}{m_{A}}\right) \frac{c_{A} c_{R} m_{R}}{d_{A}}+\frac{c_{R}^{2} m_{R}}{d_{A}}\right] .
\end{aligned}
$$

Thus, analytical explicit solutions are found which can be used for optimization of the original design parameters.

The optimization is performed for a compact passenger car with a body mass $m_{A}=1200 \mathrm{~kg}$. Then, there remain four design variables with the following reference values:

$$
\begin{aligned}
\text { wheel mass } m_{R} & =80 \mathrm{~kg}, \\
\text { body spring } c_{A} & =30000 \mathrm{~N} / \mathrm{m}, \\
\text { tyre spring } c_{R} & =320000 \mathrm{~N} / \mathrm{m}, \\
\text { shock absorber } d_{A} & =4800 \mathrm{Ns} / \mathrm{m} .
\end{aligned}
$$

This design variables are generalized quantities. For instance, the wheel mass $m_{R}$ includes not only the masses of the four wheels but also the corresponding moments of inertia of the control arms of the wheel suspensions.

For the discussion of the sensitivity with respect to the design variables, the following specific quantities are useful.

$$
\begin{aligned}
& \frac{\sigma_{a}^{2}\left(c_{A}, d_{A}\right)}{\sigma_{a}^{2} \text { reference }} \text { driving comfort by suspension strut; } \\
& \frac{\sigma_{f}^{2}\left(c_{A}, d_{A}\right)}{\sigma_{f \text { reference }}^{2}} \text { driving safety by suspension strut; } \\
& \frac{\sigma_{a}^{2}\left(c_{R}, m_{R}\right)}{\sigma_{a}^{2} \text { reference }} \text { driving comfort by axle and tyre; } \\
& \frac{\sigma_{f}^{2}\left(c_{R}, m_{R}\right)}{\sigma_{f}^{2} \text { reference }} \text { driving safety by axle and tyre. }
\end{aligned}
$$

Thus, the original four-dimensional optimization problem is reduced to two two-dimensional optimization problems which can be treated graphically very nicely (figures 4 to 7 ). For the strut parameters the following tendencies are found.

(1) Large and small damper coefficients are harmful, for medium damping comfort and safety are optimal.

(2) Small spring and damper coefficients improve the comfort considerably. However, soft springs result in large variations of the equilibrium position due to body mass variations which may only compensated by a ride-height control system. 

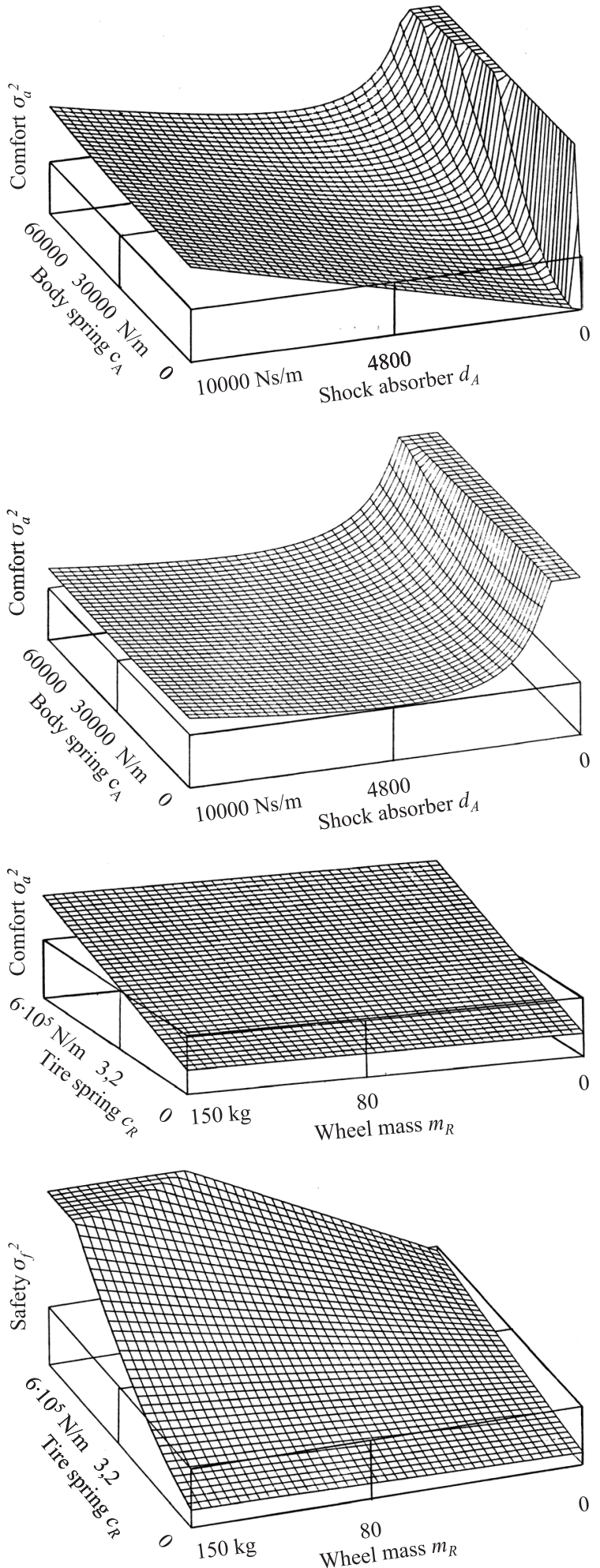

Figure 4. Driving comfort depending on strut parameters.

Figure 5. Driving safety depending on strut parameters.

Figure 6. Driving comfort depending on tyre parameters.
Figure 7. Driving safety depending on tyre parameters. 
(3) Safety is independent of the spring coefficient.

(4) The reference value of the damping coefficient yields optimal safety. Damping coefficient with optimal comfort essentially deteriorates safety. Since the efficiency of a damper decreases with time, drivers in used cars feel more comfortable, forgetting about safety.

For axle and tyre parameters also some general tendencies are observed.

(1) Comfort is independent of the tyre mass.

(2) Small tyre mass improves safety essentially. The generalized mass can be also reduced by a suitable suspension design. Nevertheless, the reductions are limited.

(3) Small tyre stiffness improves comfort and safety. Air pressure in the tires is responsible for tyre stiffness and cannot reduced essentially. Low pressure results in strong wear of the tyre and weak lateral guidance of the vehicle. Therefore, the reference value of the tyre spring coefficient is a boundary optimum.

In summary, the reference values of the suspension are close to the optimum that was determined empirically during the development of the automobile over more than a hundred years. Further progress will be achieved in the future by mechatronic system design where the stochastic methods presented will be most helpful.

In addition to the qualitative results reported in this paper, more quantitative results can be obtained for specific vehicles with more complex nonlinear models represented by the equations of motion (1) and coloured noise (19). However, for fundamental understanding of the principles of driving comfort and driving safety, a linear model with white velocity noise is most adequate.

\section{Conclusions}

Road vehicle structures are subject to random vibrations due to the unevenness of roads. A vehicle's performance with respect to vertical vibrations is assessed by the body acceleration and the wheel-load variation. Using the white noise velocity characteristic of standard roads, the standard deviation or variance respectively, of body acceleration and wheel load are evaluated explicitly in analytic form via a polynomial solution of the corresponding Lyapunov matrix equation. The performance analysis of a quarter car model representing a present-day compact car shows that the reference values are quite optimal considering the engineering constraints. Further progress will be achieved in the future by mechatronic system design where the stochastic excitation by the road remains unchanged and, therefore, stochastic structural dynamics will play an essential role.

\section{List of symbols}

A system matrix, auxiliary matrix;

a accerelation;

$\boldsymbol{a}$ vector function;

$\boldsymbol{B}$ input matrix;

c spring coefficient;

c weighting vector;

$\boldsymbol{D}$ damping matrix; 
$d \quad$ damping coefficient;

d weighting vector;

$E$ expectation;

$\boldsymbol{E}$ unit matrix;

$\boldsymbol{e}$ weighting vector;

$\boldsymbol{F}$ shape filter matrix, frequency response matrix;

$f$ number of degrees of freedom;

$\boldsymbol{G}$ gyroscopic matrix;

$g$ shape filter vector;

$\boldsymbol{H}$ shape filter matrix, Hurwitz matrix;

$H$ cofactor of Hurwitz matrix;

$\boldsymbol{h}$ excitation vector, shape filter vector;

$\boldsymbol{K}$ stiffness matrix;

$\boldsymbol{k} \quad$ Coriolis force vector;

$\boldsymbol{M}$ inertia matrix;

$m$ dimension of shape filter, mass;

$N$ matrix of nonconservative forces;

$n$ dimension of state vector;

$\boldsymbol{P}$ matrix of velocity forces, covariance matrix;

$Q \quad$ matrix of position forces, input intensity matrix;

$\boldsymbol{q}$ vector of applied forces;

$\boldsymbol{R}$ correlation matrix;

$R \quad$ Rayleigh dissipation function;

$S \quad$ two-sided power spectral density matrix;

$T$ kinetic energy;

$t$ time;

$U$ potential energy;

$u$ state excitation vector;

$\boldsymbol{v} \quad$ filter state vector;

$v \quad$ velocity;

$w$ waviness;

$\boldsymbol{x}$ state vector;

$x$ road profile coordinate;

$\boldsymbol{y}$ position vector;

$\alpha \quad$ colored noise constant;

$\beta \quad$ colored noise constant;

$\delta \quad$ Dirac function;

$\Phi$ one-sided power spectral density matrix;

$\lambda$ eigenvalue;

$\sigma \quad$ standard deviation;

$\tau \quad$ correlation time;

$\Omega$ spatial circular frequency;

$\omega$ temporal circular frequency;

$\xi \quad$ correlation distance;

$\mathrm{Z}$ spatial roughness profile;

$\zeta$ temporal roughness profile. 


\section{References}

DeRusso P M, Roy R J, Close C M, Desrochers A A 1997 State variables for engineers 2nd edn (New York: Wiley-Interscience)

Dodds C J, Robson J D 1973 The description of road surface roughness. J. Sound Vibr. 31: 175-183

Eberhard P, Schiehlen W 2004 Technische Dynamik 2nd edn (Wiesbaden: Teubner)

ISO-8608 1995 Mechanical vibration - road surface profiles - reporting of measured data. Geneva, Int. Standard Organisation

Iyengar R N 2001 Probabilistic methods in earthquake engineering (eds) H Aref, J W Phillips (Dordrecht: Kluwer) pp 457-472

Iyengar R N, Dash P K 1978 Random vibration of spacecraft structures: A review. J. Aeronaut. Soc. India 30: 1-21

Iyengar R N, Iyengar K T S 1969 A nonstationary random process model for earthquake accelerograms. Bull. Seismol. Soc. Am. 59: 1163-1188

Iyengar R N, Iyengar K T S 1970 Probabilistic response analysis to earthquake. J. Eng. Mech., ASCE 96(EM3): 207-225

Iyengar R N, Jaiswal O R 1995 Random field modelling of railway track irregularities. J. Transport Eng., ASCE 121: 303-308

Kreisselmeier G 1972 A solution of the bilinear matrix equation $A Y+Y B=-Q$. SIAM J. Appl. Math. 23: 334-338

Mucka P 2004 Road waviness and the dynamic tyre force. Int. J. Vehicle Design 36: 216-232

Mueller P C, Popp K 1979 Kovarianzanalyse von linearen Zufallsschwingungen mit zeitlich verschobenen Erregerprozessen. Z. Angew. Math. Mech. 59: T144-T146

Mueller P C, Schiehlen W 1985 Linear vibrations (Dordrecht: Martinus Nijhoff)

Mueller P C, Popp K, Schiehlen W O 1980 Berechnungsverfahren fuer stochastische Fahrzeugschwingungen. Ing. Arch. 49: 235-254

Popp K 1982 Stochastic and elastic guideway models (ed.) W O Schiehlen (Wien: Springer) pp 13-38

Popp K, Schiehlen W 1993 Fahrzeugdynamik (Stuttgart: Teubner)

Schiehlen W 1990 Multibody systems handbook (Berlin: Springer)

Schiehlen W 1997 Multibody system dynamics: Roots and perspectives. Multibody Syst. Dyn. 1: 149188

Schiehlen W 2005 Recent developments in multibody dynamics. J. Mech. Sci. Technol. 19: 227-236

Smith R A 1968 Matrix equation $X A+B X=C$. SIAM J. Appl. Math. 16: 198-201

von Scheidt J, Wunderlich R, Fellenberg B 1999 Random road surfaces and vehicle vibration (eds)

L Arkeryd, J Bergh, R Pettersson (Stuttgart: Teubner) pp 114-121 